\title{
Cold dust in three massive evolved stars in the LMC ${ }^{\star}, \star \star$
}

\author{
M. L. Boyer ${ }^{1}$, B. Sargent ${ }^{1}$, J. Th. van Loon $^{2}$, S. Srinivasan ${ }^{3}$, G. C. Clayton ${ }^{4}$, F. Kemper ${ }^{5}$, L. J. Smith ${ }^{1}$, M. Matsuura ${ }^{6,7}$, \\ Paul M. Woods ${ }^{5}$, M. Marengo ${ }^{8}$, M. Meixner ${ }^{1, \star \star \star}$, C. Engelbracht ${ }^{9}$, K. D. Gordon ${ }^{1}$, S. Hony ${ }^{10}$, R. Indebetouw ${ }^{11}$, \\ K. Misselt ${ }^{9}$, K. Okumura $^{10}$, P. Panuzzo ${ }^{10}$, D. Riebel ${ }^{12}$, J. Roman-Duval ${ }^{1}$, M. Sauvage ${ }^{10}$, and G. C. Sloan ${ }^{13}$ \\ (Affiliations are available in the online edition)
}

Received 26 March 2010 / Accepted 20 Mai 2010

\section{ABSTRACT}

\begin{abstract}
Massive evolved stars can produce large amounts of dust, and far-infrared (IR) data are essential for determining the contribution of cold dust to the total dust mass. Using Herschel, we search for cold dust in three very dusty massive evolved stars in the Large Magellanic Cloud: R71 is a luminous blue variable, HD 36402 is a Wolf-Rayet triple system, and IRAS05280-6910 is a red supergiant. We model the spectral energy distributions using radiative transfer codes and find that these three stars have mass-loss rates up to $10^{-3} M_{\odot} \mathrm{yr}^{-1}$, suggesting that high-mass stars are important contributors to the life-cycle of dust. We found far-IR excesses in two objects, but these excesses appear to be associated with ISM and star-forming regions. Cold dust $(T<100 \mathrm{~K})$ may thus not be an important contributor to the dust masses of evolved stars.
\end{abstract}

Key words. Magellanic Clouds - circumstellar matter - stars: mass-loss - stars: massive - submillimeter: stars

\section{Introduction}

Intermediate-mass asymptotic giant branch (AGB) stars are potentially the dominant dust source in the Galaxy (Gehrz 1989) and in low-metallicity environments like the Large Magellanic Cloud (LMC; Srinivasan et al. 2009; Matsuura et al. 2009) and other dwarf galaxies (Boyer et al. 2009). However, dust production in high-mass stars $\left(\gtrsim 8 M_{\odot}\right)$ remains uncertain. It has been suggested that supernovae ( $\mathrm{SNe}$ ) might be the dominant dust factory at high- $z$, since intermediate-mass stars have not yet had time to evolve into AGB stars (Morgan \& Edmunds 2003; Dwek et al. 2009). However, the amount of dust formed in SNe in the local universe is much less than required to explain the dust seen at high- $z$ (e.g., Sugerman et al. 2006; Andrews et al. 2010). It is also unclear if the dust forms before or after the SN explosion. Alternatively, Sloan et al. (2009) and Valiante et al. (2009) show that AGB stars can contribute dust at high redshifts. These studies point to a need to measure the total dust mass from all types of stars to obtain a global picture of dust evolution in galaxies.

Part of the LMC was observed with Herschel (Pilbratt et al. 2010) as part of the science demonstration program (SDP) and the Legacy program entitled HERschel Inventory of The Agents of Galaxy Evolution (HERITAGE; Meixner et al. 2010). In this letter, we describe a first look at Herschel Photodetector Array Camera and Spectrometer (PACS; Poglitsch et al. 2010) and Spectral and Photometric Imaging REceiver (SPIRE; Griffin et al. 2010) detections of 3 examples of dust-producing massive evolved stars in the LMC: the Wolf-Rayet (WR) system

\footnotetext{
* Herschel is an ESA space observatory with science instruments provided by European-led Principal Investigator consortia and with important participation from NASA.

$\star \star$ Table 2 is only available in electronic form at http: //www . aanda. org

$\star \star \star$ Visiting Scientist at Smithsonian Astrophysical Observatory, Harvard-CfA, 60 Garden St., Cambridge, MA, 02138.
}

HD 36402, the luminous blue variable (LBV) HDE 269006 (or R71), and the red supergiant (RSG) OH/IR star IRAS052806910. While such stars have been studied extensively in the midIR (e.g., Morris et al. 1999; Clark et al. 2003; Crowther 2007; Bonanos et al. 2009; van Loon et al. 2010), this study is among the first to probe them at $\lambda \gtrsim 200 \mu \mathrm{m}$.

\section{Mid- to far-infrared photometry}

We obtained PACS (100 and $160 \mu \mathrm{m})$ and SPIRE (250, 350 and $500 \mu \mathrm{m}$ ) fluxes using apertures roughly the size of the source full-width at half-maximum and sky apertures avoiding regions of high background (Table 1). We also performed aperture photometry on the Multiband Imaging Photometer for Spitzer (MIPS; Rieke et al. 2004) images from the Surveying The Agents of Galaxy Evolution Spitzer Legacy program (SAGE; Meixner et al. 2006).

In Sect. 3 we examine the optical to far-IR spectral energy distributions (SEDs). Optical $U B V I$ and near-IR $J H K$ photometry are from the Magellanic Clouds Photometric Survey (Zaritsky et al. 1997) and the Two Micron All-Sky Survey (Skrutskie et al. 2006), via the SAGE catalog. $J, K$, and $L^{\prime}$ photometry of IRAS05280-6910 are from van Loon et al. (2005). I-band photometry for HD 36402 is from the Deep NearInfrared Southern Sky Survey (Epchtein et al. 1997). InfraRed Array Camera (IRAC; Fazio et al. 2004) 3.6-8.0 $\mu \mathrm{m}$ photometry is from the SAGE catalog. Spectra from the InfraRed Spectrograph (IRS; 5.2-38 $\mu \mathrm{m}$; Houck et al. 2004) and MIPS spectra (MIPS-SED; 52-97 $\mu \mathrm{m}$ ) are also included (e.g., SAGESpec; Kemper et al. 2010; van Loon et al. 2010). We correct for extinction using the extinction map from Schlegel et al. (1998), assuming the stars lie midway the LMC contribution: $A_{\mathrm{V}} \approx$ $1 \mathrm{mag}$ for HD 36402 and IRS05280-6910 and $A_{\mathrm{V}} \approx 0.4 \mathrm{mag}$ for R71. 
Table 1. Target information and Herschel flux densities.

\begin{tabular}{llllllll}
\hline \hline Name & RA, Dec (J2000) & Type & & & $F_{v}(\mathrm{mJy})$ \\
& & & $100 \mu \mathrm{m}$ & $160 \mu \mathrm{m}$ & $250 \mu \mathrm{m}$ & $350 \mu \mathrm{m}$ & $500 \mu \mathrm{m}$ \\
\hline R71 & $05^{\mathrm{h}} 02^{\mathrm{m}} 07.39^{\mathrm{s}},-71^{\circ} 20^{\prime} 13.1^{\prime \prime}$ & LBV & $447 \pm 8$ & $105 \pm 10$ & $50 \pm 6$ & $49 \pm 13$ & $27 \pm 9$ \\
IRAS05280-6910 & $05^{\mathrm{h}} 27^{\mathrm{m}} 40.11^{\mathrm{s}},-69^{\circ} 08^{\prime} 04.5^{\prime \prime}$ & RSG, OH/IR & $\ldots$ & $\ldots$ & $205 \pm 8$ & $46 \pm 13$ & $<11 \pm 10$ \\
HD 36402 & $05^{\mathrm{h}} 26^{\mathrm{m}} 03.94^{\mathrm{s}},-67^{\circ} 29^{\prime} 57.9^{\prime \prime}$ & WC4(+O?)+O8I & $\ldots$ & $\ldots$ & $\ldots$ & $\ldots$ & $\ldots$ \\
HD 36402 IR1/YSO2 & $05^{\mathrm{h}} 26^{\mathrm{m}} 02.99^{\mathrm{s}},-67^{\circ} 29^{\prime} 57.7^{\prime \prime}$ & YSO? & $531 \pm 10$ & $872 \pm 33$ & $677 \pm 33$ & $<431 \pm 23$ & $<132 \pm 21$ \\
\hline
\end{tabular}

Notes. Quoted uncertainties are $1 \sigma$. Upper limits are either below the $3 \sigma$ detection level or include substantial flux from an adjacent unresolved source. IRAS05280-6910 does not fall within the PACS coverage. The WR star itself is not resolved in Herschel images; it is severely blended with an IR source (IR1/YSO2). The 350 and $500 \mu \mathrm{m}$ fluxes of IR1/YSO2 include a nearby YSO (N51-YSO1), which is unresolved from IR1/YSO2 at these wavelengths. Aperture corrections for PACS and SPIRE were estimated using the current point-spread functions, and are roughly 1.4 and 1.2, respectively. None of the sources are heavily affect by high and/or variable backgrounds (Fig. 1).

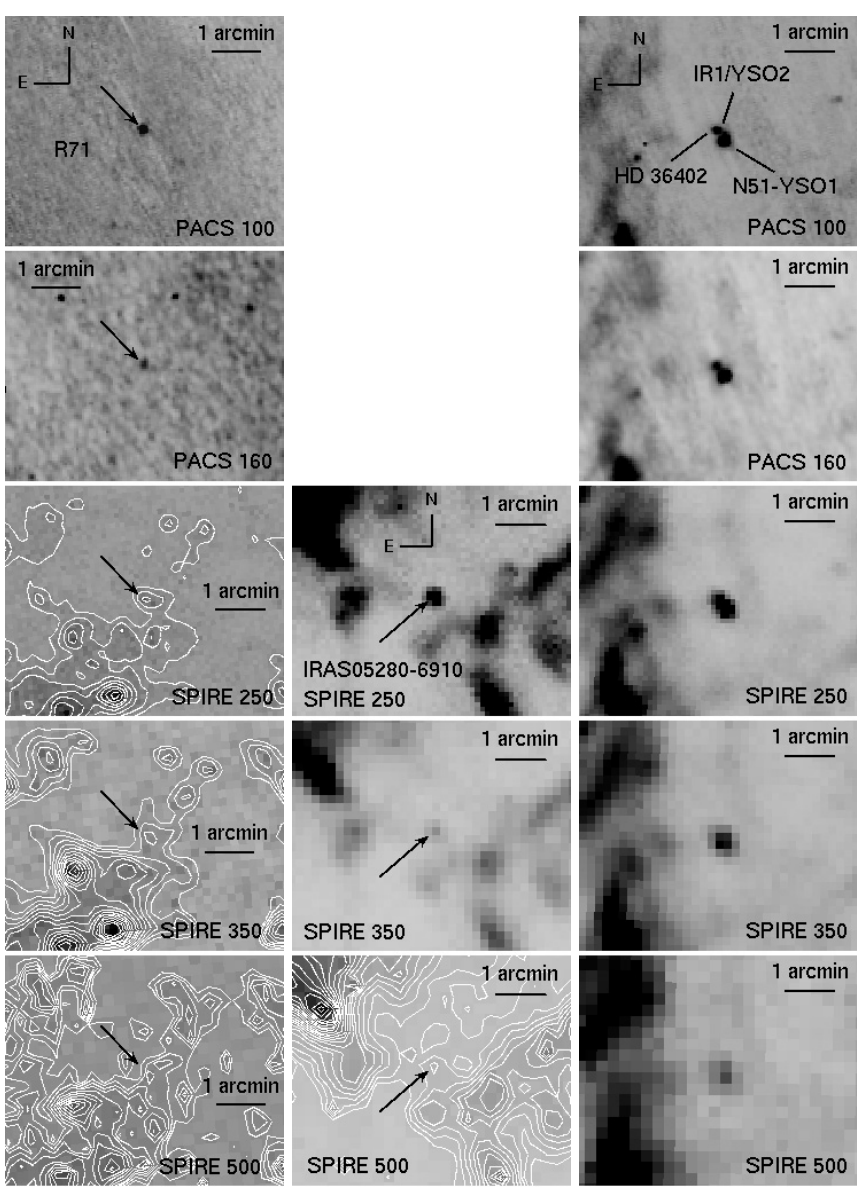

Fig. 1. Herschel images of R71 (left panels), IRAS05280-6910 (middle panels), and HD 36402 (right panels, also see Fig. 3). Contours on a linear scale are included where it is difficult to see the detection.

\section{The IR nature of detected sources}

The vast majority of point-sources detected in the LMC SDP data appear to be young stellar objects (YSOs) or background galaxies (Sewiło et al. 2010); AGB stars were not detected. Here, we describe the Herschel observations of three very dusty massive stars (Fig. 1).

\section{1. $R 71$}

R71 (Fig. 1) is an LBV with $M_{\text {birth }} \approx 40 M_{\odot}$ (Lennon et al. 1993), and is currently experiencing an unprecedented eruption that began in 2005. One month prior to the SDP observations (Oct. 2009), it showed a $\sim 2$ mag increase in visual brightness and the $V$-band light curve was just beginning to plateau (Szczygiel et al. 2010). Van Loon et al. (2010) find a lack of cold dust in R71, as indicated by Spitzer data $\left(T_{\mathrm{d}} \lesssim 100 \mathrm{~K}\right)$. The Spitzer data were acquired just prior to the current outburst, and the Herschel data presented here were obtained at nearmaximum. However, the PACS and MIPS points appear consistent with each other, such that a model fit to the optical to MIPS data also agrees with the PACS points. This indicates that the increased emission from the photosphere has not yet significantly affected the $100-160 \mu \mathrm{m}$ flux. Note that the MIPS $160 \mu \mathrm{m}$ point is an upper limit.

IRS and ISO spectra of R71 show strong PAH, crystalline forsterite and enstatite features (Voors et al. 1999; Morris et al. 2008; Buchanan et al. 2009; Waters 2010). A $10.5 \mu \mathrm{m}$ amorphous silicate feature indicates dominantly oxygen-rich (O-rich) chemistry. Morris et al. (2008) and Voors et al. (1999) speculate that the dust was formed during a prior RSG phase.

Figure 2 shows R71's SED. Ultraviolet spectra (Blair et al. 2009) are consistent with the stellar component included in our model. We fit the SED with a 2-DUST model (online Table 2; Ueta \& Meixner 2003), adopting spherical symmetry and grain properties of the dominant species: amorphous silicate. Three dust components are visible, including a previously unknown excess visible in the SPIRE data at 250, 350 and $500 \mu \mathrm{m}(8$, 3.8 , and $3 \sigma$ detections, respectively). The two warmer components are modeled by the 2-DUST code using two concentric dust shells. We assume the gas-to-dust ratio for the LMC is $\psi=300$ (cf. Meixner et al. 2010), yielding $\dot{M} \sim 10^{-6} M_{\odot} \mathrm{yr}^{-1}$ for the inner dust shell and $\dot{M} \sim 10^{-3} M_{\odot} \mathrm{yr}^{-1}$ for the outer shell, assuming $v_{\text {wind }}=10 \mathrm{~km} \mathrm{~s}^{-1}$, consistent with the wind speed of a shell ejected during an RSG phase. Stahl et al. (1986) find $v \approx 160 \mathrm{~km} \mathrm{~s}^{-1}$ from the $\mathrm{H} \alpha$ line profile, indicating the MLR for the inner shell could be an order of magnitude higher. A DUSTY model (Nenkova et al. 1999) fits the SED equally well and estimates a MLR that is the 2-DUST outer shell value.

We fit the far-IR excess with a modified blackbody: $F_{\lambda} \propto B_{\lambda}\left(T_{\mathrm{d}}\right)\left(1-\mathrm{e}^{-\tau_{\lambda}}\right)$, where $B_{\lambda}\left(T_{\mathrm{d}}\right)$ is the Planck function at temperature $T_{\mathrm{d}}, \tau_{\lambda}$ is the optical depth, and $\tau_{\lambda} \propto \lambda^{-\beta}$. Here, we use $\beta=1.5$. The resulting dust temperature is $9 \pm 1 \mathrm{~K}$, which is extremely low compared to the expected temperature of outer shells in evolved stars ( $30 \mathrm{~K}$; Speck et al. 2000), and is instead consistent with temperatures of dense ISM dust clouds (see Sect. 4).

\subsection{IRAS05280-6910}

IRAS05280-6910 is an RSG OH/IR star. It is detected in all Spitzer and Herschel bands up to $350 \mu \mathrm{m}$ (>3.5 $\sigma$; Figs. 1 and 2$)$. The star is heavily extinguished in the optical and near-IR, and the 10 and $18 \mu \mathrm{m}$ silicate features are seen in absorption in the IRS spectrum (Kemper et al. 2010). Van Loon et al. (2010) find no indication of dust colder than $\sim 100 \mathrm{~K}$ in the MIPS data. 


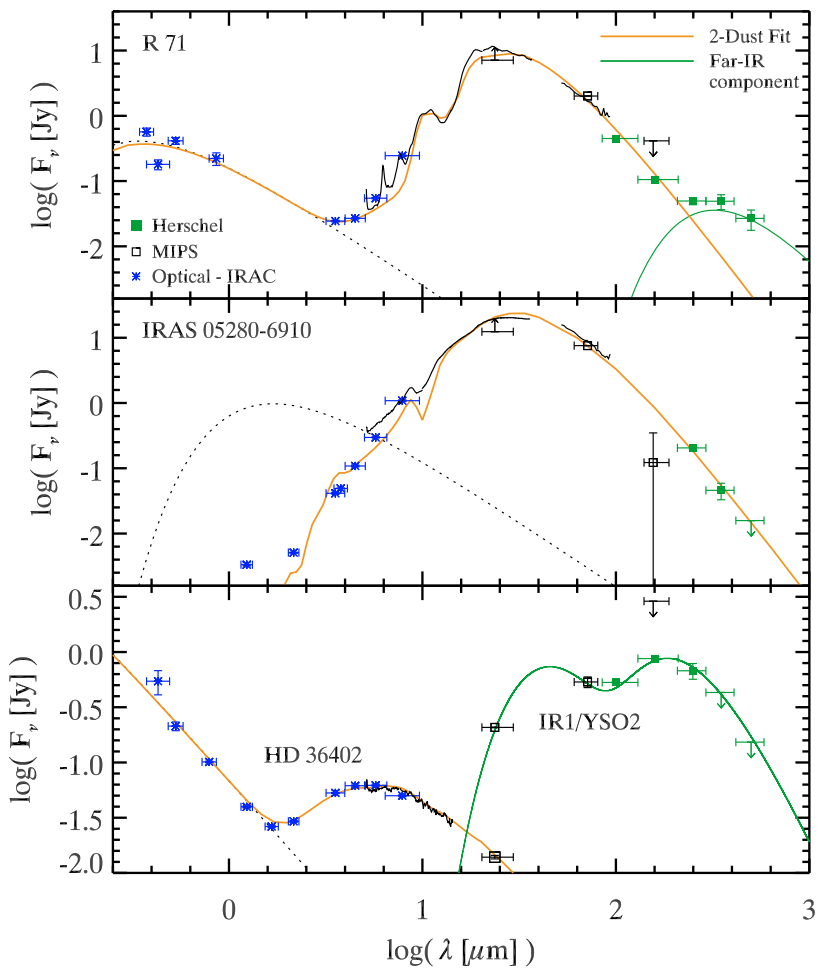

Fig. 2. SEDs of R71 (top), IRAS05280-6910 (middle) and HD 36402 (bottom), fit to 2-DUST models (orange lines). Far-IR excesses are fit to modified blackbodies (green lines). The IRS and MIPS-SED spectra are shown in black and the stellar components are the dotted lines. In the upper two panels, the $24 \mu \mathrm{m}$ points are saturated (lower limits). In the bottom panel, the bright far-IR emission originates from IR1/YSO2; the WR system is detected in PACS, but unresolved from IR1. See text.

We fit the SED of IRAS05280-6910 using 2-DUST (flattened geometry) and DUSTY (spherical symmetry). Both models fit the SED reasonably well (only the 2-DUST model is shown in Fig. 2), but predict stronger absorption in the $10 \mu \mathrm{m}$ silicate feature than is seen in the IRS spectrum, even when including grains as large as $1 \mu \mathrm{m}$ (increasing $a_{0}$ suppresses the silicate feature). This discrepancy may be due to the inclusion in the slit of a nearby RSG (WOH G347), which is too faint to contribute to the SED at other wavelengths, but shows emission near $10 \mu \mathrm{m}$ with enough flux to veil the silicate absorption by the necessary amount (van Loon et al. 2005). Based on both model fits and assuming an outflow velocity of $\approx 20 \mathrm{~km} \mathrm{~s}^{-1}$ (as measured from maser emission; Marshall et al. 2004), the mass-loss rate (MLR) was $\dot{M}=2-8 \times 10^{-3} M_{\odot} \mathrm{yr}^{-1}$ when the dust was produced (online Table 2). There is no evidence of excess emission at $\lambda>100 \mu \mathrm{m}$ in the SDP data, implying there is no significant contribution from cold $(\ll 100 \mathrm{~K})$ and/or large $(\mu \mathrm{m}-\mathrm{mm}$ size $)$ grains.

\section{3. $H D 36402$}

HD 36402 (Fig. 3) is a WR star that is part of a triple system (Moffat et al. 1990). It is the reddest LMC WR star studied by Bonanos et al. (2009) in IRAC, potentially due to dust formed in colliding stellar winds (Crowther 2007). HD 36402 is detected by PACS (Fig. 3b), which might indicate the presence of some cool dust. However, the system is almost totally unresolved from the stronger far-IR emission immediately to the west (Figs. 1 and $3 b$ ), which appears to originate from a nearby molecular cloud, visible in $\mathrm{H} \alpha$ (Fig. 3; Dopita et al. 1994; Chu et al. 2005). We refer to this emission as HD 36402 IR1.

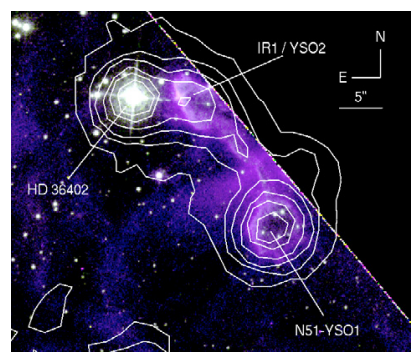

(a)

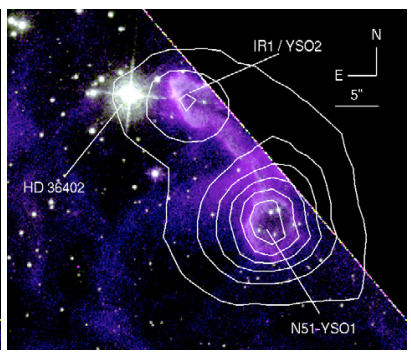

(b)
Fig. 3. Composite HST/WFPC2 image of HD 36402 in $\mathrm{H} \alpha$ and [S II] (purple). The contours represent a) IRAC $8 \mu \mathrm{m}$; and b) PACS $100 \mu \mathrm{m}$.

The IRS spectrum of HD 36402 from SAGE-Spec is featureless and reminiscent of $\mathrm{R}$ Coronae Borealis stars, which are C-rich (no silicate dust) and hydrogen-deficient (no PAHs). The continuum emission is thought to be due to amorphous carbon dust (e.g., Kraemer et al. 2005). The SED (Fig. 2) shows a stellar component and a component from a detached, dusty shell $(\sim 2-24 \mu \mathrm{m})$. The far-IR SED is dominated by IR1, so an accurate fit to the WR system in the far-IR is not possible with this dataset. In the lowest resolution images (MIPS 160, SPIRE 350 and $500 \mu \mathrm{m}$ ), IR1 is also unresolved from N51-YSO1 (upper limits in Fig. 2), further complicating the SED. A 2-DUST model (Fig. 2, orange line) gives $\dot{M}=7 \times 10^{-6} M_{\odot} \mathrm{yr}^{-1}$.

What is the nature of IR1? Based on its IRAC colors, Chu et al. (2005) identify it as a YSO (N51-YSO2). We have attempted to fit a two-component modified blackbody $(\beta=2.0)$ to the IR1 SED to check if it instead originates from dust heated directly by the radiation emanating from HD 36402. These fits yield a dust temperature of $64 \mathrm{~K}$, with $15 \mathrm{~K}$ dust in its wake, consistent with this scenario. However, a temperature of $64 \mathrm{~K}$ places the molecular cloud $\sim 0.3 \pm 0.1 \mathrm{pc}$ from the WR system, assuming typical luminosities of a WC4 star and two O star companions. The high-resolution $\mathrm{H} \alpha$ image (Fig. 3) puts the molecular cloud at least $1.2 \mathrm{pc}$ away from the WR system, assuming a distance of $50 \mathrm{kpc}$ to the LMC (Schaefer 2008). It thus seems likely that the far-IR emission of IR1 is indeed related to a YSO embedded in the molecular cloud rather than to direct heating from HD 36402. In the HST image (Fig. 3), the cloud appears brightest in the region adjacent to HD 36402, which may indicate some interaction between the two. If this is indeed the case, it is plausible that the formation of YSO2 was triggered by HD 36402.

\section{Implications}

The new far-IR Herschel data have allowed us to take the first steps in assessing the contribution of cold dust to the total dust mass in 3 massive stars. For the RSG IRAS05280-6910, the models give a total dust mass of $\approx 0.3 M_{\odot}$. This dust mass is extremely large for an RSG star; indeed it is also highly uncertain, as it is computed from the dust density distribution and the grain composition and size distribution, which introduces many degeneracies. By adjusting these parameters, we can reduce the dust mass by at least $60 \%$, possibly more. The MLR (online Table 2), while still uncertain, is a more reliable quantity and suggests the star has entered a necessarily-brief phase of extreme mass loss. The large mass found using our simple, preliminary models may indicate a complicated geometry with a preferential viewing angle requiring more extensive modeling, or perhaps the RSG is embedded in a dusty cloud. Whatever the total envelope mass, a lack of excess far-IR emission over the model indicates that ancient, cold dust does not contribute significantly. Dust at 
$50 \mathrm{~K}$, for instance, must contribute less than $10 \%$ of the dust mass implied by the model fit to explain this lack of far-IR excess.

The LBV R71 shows three dust components. A component emitting at $\sim 10-100 \mu \mathrm{m}$ resembles RSG dust. A second component dominates at $3-10 \mu \mathrm{m}$. Its temperature $\left(T_{\text {in }}=490 \mathrm{~K}\right)$ suggests it formed $<50$ yrs ago, assuming $v \sim 100 \mathrm{~km} \mathrm{~s}^{-1}$ and $T_{0}=1000 \mathrm{~K}$, perhaps during the 1970 s outburst (Wolf \& Zickgraf 1986). The third, more tentative dust component is visible at $\lambda>250 \mu \mathrm{m}$. If this feature corresponds to cold circumstellar dust, the implied dust mass is $M_{\text {dust }} \gtrsim 10^{-1} M_{\odot}$, following Evans et al. (2003) and assuming the absorption coefficient, $\kappa(500 \mu \mathrm{m})$, is $20 \mathrm{~cm}^{2} \mathrm{~g}^{-1}$ (Ossenkopf \& Henning 1994). This is far too much dust for a star of this mass; together with its very cold temperature $(9 \mathrm{~K})$, this high mass suggests that the farIR emission is pre-existing ISM dust swept up by stellar winds and/or is ISM dust along the line-of-sight. Indeed, the contours in Fig. 1 appear to show diffuse emission at the position of R71 at the longest wavelengths. If the far-IR emission instead originates from the circumstellar envelope, then very large grains similar to the cm-sized grains in the Egg Nebula (Jura et al. 2000) might explain the far-IR emission without requiring the implied large dust mass and cold temperature. Follow-up spectroscopy or deeper SPIRE imaging may help uncover the nature of the far-IR emission.

The WR system, HD 36402, seems to be forming dust in colliding winds, which is too warm to emit much at far-IR wavelengths. The apparent far-IR emission from HD 36402 (Fig. 3b) is unfortunately totally overwhelmed by far-IR emission originating from a nearby molecular cloud. Assuming $\kappa(70 \mu \mathrm{m}) \approx$ $140 \mathrm{~cm}^{2} \mathrm{~g}^{-1}$ and $\kappa(500 \mu \mathrm{m}) \approx 20 \mathrm{~cm}^{2} \mathrm{~g}^{-1}$ (Ossenkopf \& Henning 1994), we find $M_{\text {dust }}^{63 \mathrm{~K}}=1.0( \pm 0.3) \times 10^{-2} M_{\odot}$ and $M_{\text {dust }}^{15 \mathrm{~K}}=1.5 \pm 0.4 M_{\odot}$ of dust in the molecular cloud.

Stellar evolution models, while still uncertain, show that massive stars like these eventually explode as SNe. Due to its proximity to a molecular cloud, the HD 36402 remnant may resemble the SN remnant N49, which has swept up $0.2 M_{\odot}$ of dust from the ISM (van Loon et al. 2010; Otsuka et al. 2010). The absence of strong evidence for very large grains in R71 and IRAS05280-6910 does not raise the prospects of RSG dust surviving a SN blast. Regardless of their fates, the dust masses in these 3 stars are quite large, compared with the dust mass found in a typical AGB star, showing that high-mass stars are important contributors to the life-cycle of dust even in low-metallicity environments like the LMC. However, we emphasize that we do not find strong evidence for cold dust and/or large grains in any of the three objects discussed here, except where it is certain (HD 36402) or likely (R71) to be of interstellar origin and not synthesized by the object itself. These observations indicate that far-IR data of a much larger sample of luminous evolved stars in both Magellanic Clouds will be obtained in the full HERITAGE dataset, from which we expect clearer patterns to emerge.

Acknowledgements. We thank the referee for his or her helpful comments. This publication includes observations made with the NASA/ESA $H S T$, and obtained from the Hubble Legacy Archive, which is a collaboration between the Space Telescope Science Institute (STScI/NASA), the Space Telescope European Coordinating Facility (ST-ECF/ESA) and the Canadian Astronomy Data Centre (CADC/NRC/CSA). We acknowledge financial support from the NASA Herschel Science Center, JPL contract \# 1381522. We thank the contributions and support from the European Space Agency (ESA), the PACS and SPIRE teams, the Herschel Science Center and the NASA Herschel Science Center (esp. A. Barbar and K. Xu) and the PACS/SPIRE instrument control center at CEASaclay, which made this work possible.

\section{References}

Andrews, J. E., Gallagher, J. S., Clayton, G. C., et al. 2010, ApJ, 715, 541 Blair, W. P., Oliveira, C., Lamassa, S., et al. 2009, PASP, 121, 634 Bonanos, A. Z., Massa, D. L., Sewilo, M., et al. 2009, AJ, 138, 1003 Boyer, M. L., Skillman, E. D., van Loon, J. Th., Gehrz, R. D., \& Woodward, C. E. 2009, ApJ, 697, 1993

Buchanan, C. L., Kastner, J. H., Hrivnak, B. J., \& Sahai, R. 2009, AJ, 138, 1597 Chu, Y., Gruendl, R. A., Chen, C., et al. 2005, ApJ, 634, L189

Clark, J. S., Egan, M. P., Crowther, P. A., et al. 2003, A\&A, 412, 185 Crowther, P. A. 2007, ARA\&A, 45, 177

Dopita, M. A., Bell, J. F., Chu, Y., \& Lozinskaya, T. A. 1994, ApJS, 93, 455 Draine, B. T., \& Lee, H. M. 1984, ApJ, 285, 89

Dwek, E., Galliano, F., \& Jones, A. 2009, ASP Conf. Ser., 414, 183 Epchtein, N., de Batz, B., Capoani, L., et al. 1997, The Messenger, 87, 27 Evans, A., Stickel, M., van Loon, J. Th., et al. 2003, A\&A, 408, L9 Fazio, G. G., Hora, J. L., Allen, L. E., et al. 2004, ApJS, 154, 10 Gehrz, R. D. 1989, in Interstellar Dust, ed. L. J. Allamandola, \& A. G. G. M. Tielens, IAU Symp., 135, 445

Griffin, M. J., et al. 2010, A\&A, 518, L3

Houck, J. R., Roellig, T. L., van Cleve, J., et al. 2004, ApJS, 154, 18 Jura, M., Turner, J. L., Van Dyk, S., \& Knapp, G. R. 2000, ApJ, 528, L105 Kemper, F., Woods, P. M., Antoniou, V., et al. 2010, PASP, 122, 683 Kim, S., Martin, P. G., \& Hendry, P. D. 1994, ApJ, 422, 164

Kraemer, K., Sloan, G., Wood, P. R., Price, S., \& Egan, M. 2005, ApJ, 631, L147 Lennon, D. J., Wobig, D., Kudritzki, R., \& Stahl, O. 1993, Space Sci. Rev., 66, 207

Marshall, J. R., van Loon, J. Th., Matsuura, M., et al. 2004, MNRAS, 355, 1348 Matsuura, M., Barlow, M. J., Zijlstra, A. A., et al. 2009, MNRAS, 396, 918 Meixner, M., Gordon, K. D., Indebetouw, R., et al. 2006, AJ, 132, 2268 Meixner, M., et al. 2010, A\&A, 518, L71

Moffat, A. F. J., Niemela, V. S., \& Marraco, H. G. 1990, ApJ, 348, 232 Morgan, H. L., \& Edmunds, M. G. 2003, MNRAS, 343, 427

Morris, P., Bouwman, J., Crowther, P., et al. 1999, in The Universe as Seen by ISO, ed. P. Cox, \& M. Kessler, ESA SP, 427, 247

Morris, P., \& Spitzer WRRINGS Team. 2008, in IAU Symp. 250, ed. F. Bresolin, P. A. Crowther, \& J. Puls, 361

Nenkova, M., Ivezic, Z., \& Elitzur, M. 1999, LPI Contributions, 969, 20

Ossenkopf, V., \& Henning, T. 1994, A\&A, 291, 943

Ossenkopf, V., Henning, T., \& Mathis, J. S. 1992, A\&A, 261, 567

Otsuka, M., et al. 2010, A\&A, 518, L139

Pilbratt, G. L., et al. 2010, A\&A, 518, L1

Poglitsch, A., et al. 2010, A\&A, 518, L2

Rieke, G. H., Young, E. T., Engelbracht, C. W., et al. 2004, ApJS, 154, 25 Schaefer, B. E. 2008, AJ, 135, 112

Schlegel, D. J., Finkbeiner, D. P., \& Davis, M. 1998, ApJ, 500, 525

Sewiło, M., et al. 2010, A\&A, 518, L73

Skrutskie, M. F., Cutri, R. M., Stiening, R., et al. 2006, AJ, 131, 1163

Sloan, G. C., Matsuura, M., Zijlstra, A. A., et al. 2009, Science, 323, 353

Speck, A. K., Meixner, M., \& Knapp, G. R. 2000, ApJ, 545, L145

Srinivasan, S., Meixner, M., Leitherer, C., et al. 2009, AJ, 137, 4810

Stahl, O., Wol, B., de Groot, M., \& Leitherer, C. 1986, in Luminous Stars and Associations in Galaxies, ed. C. W. H. de Loore, A. J. Willis, \& P. Laskarides, IAU Symp., 116, 247

Sugerman, B. E. K. Ercolano, B., Barlow, M. J., et al. 2006, Science, 313, 196 Szczygiel, D. M., Stanek, K. Z., et al. 2010, ApJ, 140, 14

Ueta, T., \& Meixner, M. 2003, ApJ, 586, 1338

Valiante, R., Schneider, R., Bianchi, S., \& Andersen, A. C. 2009, MNRAS, 397, 1661

van Loon, J. Th., Marshall, J. R., \& Zijlstra, A. A. 2005, A\&A, 442, 597

van Loon, J. Th., Oliveira, J. M., Gordon, K. D., et al. 2010, AJ, 139, 68

Voors, R. H. M., Waters, L. B. F. M., Morris, P. W., et al. 1999, A\&A, 341, L67

Waters, L. B. F. M. 2010, in Hot and Cool: Bridging Gaps in Massive Star Evolution, ed. C. Leitherer, Ph. D. Bennett, P. W. Morris, \& J. Th. van Loon, ASP Conf. Ser., in press,

Willis, A. J., Crowther, P. A., Fullerton, A. W., et al. 2004, ApJS, 154, 651

Wolf, B., \& Zickgraf, F. J. 1986, A\&A, 164, 435

Zaritsky, D., Harris, J., \& Thompson, I. 1997, AJ, 114, 1002

Zubko, V. G., Mennella, V., Colangeli, L., \& Bussoletti, E. 1996, MNRAS, 282, 1321

Page 5 is available in the electronic edition of the journal at http://www . aanda. org 
Table 2. SED fit parameters and results.

\begin{tabular}{llllllllll}
\hline \hline & $T_{*}$ & $L_{*}$ & $\begin{array}{l}T_{\text {in }} \\
\left(10^{4} \mathrm{~K}\right)\end{array}$ & $\begin{array}{l}R_{*} \\
\left(10^{5} L_{\odot}\right)\end{array}$ & $\begin{array}{l}R_{\text {out }} / R_{\text {in }} \\
(\mathrm{K})\end{array}$ & $\begin{array}{l}R_{\text {in }} \\
(\mathrm{km})\end{array}$ & $\begin{array}{l}\dot{M}_{\text {total }} \\
\left.\left(M_{\odot} \mathrm{yr}\right)^{-1}\right)\end{array}$ & $\begin{array}{l}T_{\text {dust }}^{\text {sub-mm }} \\
(\mathrm{K})\end{array}$ & $\begin{array}{l}M_{\text {dust }}^{\text {sub-mm }} \\
\left(M_{\odot}\right)\end{array}$ \\
\hline R71 (inner/outer shell) & 1.5 & 4.6 & $490 / 120$ & 100 & $2 / 1.6$ & $\sim 10^{11} / 10^{12}$ & $\sim 10^{-6} / \sim 10^{-4}$ & $9 \pm 1$ & $\gtrsim 10^{-1}$ \\
IRAS05280-6910 & 0.3 & 2.2 & 250 & 1700 & 30 & $\sim 10^{11}$ & $\sim 10^{-3}$ & $\ldots$ & $\ldots$ \\
HD 36402 & 18 & 4.6 & 960 & 15 & 300 & $\sim 10^{10}$ & $7 \times 10^{-6}$ & $\ldots$ & $\ldots$ \\
HD 36402 IR1 (YSO2) & $\ldots$ & $\ldots$ & $\ldots$ & $\ldots$ & $\ldots$ & $\ldots$ & $\ldots$ & $64 / 15( \pm 1)$ & $10^{-12} />1.5 \pm 0.4$ \\
\hline
\end{tabular}

Notes. Fitting results from 2-DUST. DUSTY fits for R71 and IRAS05280-6910 estimate outer shell MLRs $2 \times$ to $4 \times$ less than the 2-DUST results. IR1/YSO2 was fit with two modified blackbodies (64 K and $15 \mathrm{~K}$ ). For R71, we use O-deficient silicate optical constants (Ossenkopf et al. 1992) at long wavelengths and astronomical silicate optical constants (Draine \& Lee 1984) at $\lambda<0.18 \mu \mathrm{m}$. For IRAS05280-6910, we use Ossenkopf et al. (1992) O-deficient silicates and for HD 36402, we use amorphous carbon grains from Zubko et al. (1996). The 2-DUST models use a KMH grain size distribution (Kim et al. 1994) with $a_{\min }=0.01 \mu \mathrm{m}$ and $a_{0}=0.1 \mu \mathrm{m}\left(a_{0}=1 \mu \mathrm{m}\right.$ for IRAS05280-6910). Stellar parameters for R71 are consistent with those from Lennon et al. (1993). $T_{\text {dust }}^{\text {sub }}$ is is the temperature of the coldest dust component. For IRAS5280-6910, $v_{\text {wind }}$ is measured from the maser emission (20 km s${ }^{-1}$; Marshall et al. 2004). For R71, we use $10 \mathrm{~km} \mathrm{~s}^{-1}$, but the velocity measured from the H $\alpha$ profile indicates the inner shell velocity may be $>10 \times$ larger (Stahl et al. 1986). For HD36402, a typical velocity for a WC4 star is assumed (3000 $\mathrm{km} \mathrm{s}^{-1}$; Willis et al. 2004).

1 Space Telescope Science Institute, 3700 San Martin Drive, Baltimore, MD 21218, USA

e-mail: mboyer@stsci.edu

2 School of Physical \& Geographical Sciences, Lennard-Jones Laboratories, Keele University, Staffordshire ST5 5BG, UK

3 Institut d'Astrophysique de Paris, CNRS UPR 341, 98bis, Boulevard Arago, 75014 Paris, France

${ }^{4}$ Louisiana State University, Department of Physics \& Astronomy, 233-A Nicholson Hall, Tower Dr., Baton Rouge, LA 70803, USA

5 Jodrell Bank Centre for Astrophysics, Alan Turing Building, School of Physics and Astronomy, University of Manchester, Oxford Road, Manchester M13 9PL, UK

${ }^{6}$ Department of Physics and Astronomy, University College London, Gower Street, London WC1E 6BT, UK
7 Mullard Space Science Laboratory, University College London, Holmbury St. Mary, Dorking, Surrey RH5 6NT, UK

8 Department of Physics and Astronomy, Iowa State University, Ames, IA, 50011, USA

9 Steward Observatory, University of Arizona, 933 North Cherry Ave., Tucson, AZ 85721, USA

10 CEA, Laboratoire AIM, Irfu/SAp, Orme des Merisiers, 91191 Gif-sur-Yvette, France

11 National Radio Astronomy Observatory, Department of Astronomy, University of Virginia, PO Box 3818, Charlottesville, VA 22903 USA

12 Johns Hopkins University, Department of Physics and Astronomy, Homewood Campus, Baltimore, MD 21218, USA

13 Department of Astronomy, Cornell University, Ithaca, NY 14853, USA 